\title{
Solving the VerifyThis 2012 challenges with VeriFast
}

\author{
Bart Jacobs, Jan Smans, and Frank Piessens \\ iMinds-DistriNet, KU Leuven, 3001 Leuven, Belgium \\ Received: date / Revised version: date
}

\begin{abstract}
We describe our experience solving the VerifyThis 2012 challenges with our program verification tool VeriFast, including detailed explanations of our solutions. We also describe some alternative solutions that we developed after the competition.

VeriFast is a modular verifier that takes Java or $\mathrm{C}$ source code annotated with function/method specifications written in a variant of separation logic, and verifies that the code complies with the annotations through symbolic execution.
\end{abstract}

\section{Introduction}

The first two authors of this article participated as the VeriFast team in the VerifyThis 2012 competition [6] held during the Formal Methods 2012 conference on 30 August 2012 in Paris, France. We used the VeriFast program verification tool [12], a research prototype that we have been developing since 2008. It is a modular verifier that takes Java or C source code annotated with function/method specifications written in a variant of separation logic, and verifies that the code complies with the annotations through symbolic execution.

In this article, we describe our solutions to the competition challenges. In the process of explaining our solutions, we describe several VeriFast features that we have not yet described in earlier published work, including partial fixpoint function applications, parameterized lemma function types, and quantifiers.

VeriFast distributions for Windows, MacOS X, and Linux can be downloaded from the VeriFast website [7]. The tool ships with a test suite that includes the solutions described here in the directory examples/fm2012.

While a tutorial and other materials on VeriFast are available via the website, this article intends to be accessible to readers new to VeriFast.
The remainder of this article is structured as follows. In Section 2, we provide an overview of the VeriFast approach. In Sections 3-5, we describe our solutions to Challenges 1-3, respectively. We offer a conclusion in Section 6.

\section{Background: The VeriFast Approach}

In this section, we give an overview of the VeriFast approach. For concreteness, we describe the approach as applied to the $\mathrm{C}$ programming language; most concepts carry over straightforwardly to the Java case.

VeriFast performs modular verification: each function is verified in isolation, using only the contracts, but not the bodies, of other functions. Function contracts are expressed in a variant of separation logic [13,10], a logic for reasoning about pointer-manipulating imperative programs. Separation logic is an extension of Hoare logic [5] where assertions are interpreted with respect to a store (which maps program variables to values) and a heap (a partial function from allocated addresses to values) rather than just a store. It introduces a number of additional operators into the syntax of assertions, the most important of which are emp, stating that the heap's domain is empty; the points-to assertion $\ell \mapsto v$, stating that the heap contains exactly the mapping of address $\ell$ to value $v$ and is otherwise empty; and the separating conjunction $P * Q$, stating that the heap can be split into two disjoint subheaps such that assertion $P$ holds for one and assertion $Q$ holds for the other. The proof rule for heap mutation then becomes

$$
\left\{\ell \mapsto{ }_{-}\right\}[\ell]:=v\{\ell \mapsto v\}
$$

where $\ell \mapsto{ }_{-}$is shorthand for $\exists v . \ell \mapsto v$, and $[\ell]:=v$ is the command that assigns the value $v$ to the heap cell at address $\ell$. This proof rule is applicable only if the heap contains exactly the heap cell $\ell$ and no others. Of 
course, in general, the heap contains multiple heap cells; to accomodate this, the proof rule can be lifted to larger heaps using the frame rule:

$$
\frac{\{P\} c\{Q\}}{\{P * R\} c\{Q * R\}} \text { where freevars }(R) \cap \operatorname{modifies}(c)=\emptyset
$$

This rule states that if a command $c$ runs without failure when started in a state satisfying assertion $P$ and each post-state satisfies assertion $Q$, then it will also run without failure if started in a state obtained by enlarging the heap with a subheap that satisfies assertion $R$, and furthermore the command does not modify this subheap. (The side condition states that $R$ does not mention any program variables modified by $c$. Note that heap locations (looked up in the heap) are not considered to be program variables (looked up in the store).)

Function contracts for functions that manipulate data structures may hide the internal shape of the data structure by using an abstract separation logic predicate [11], which is essentially a named, parameterized separation logic assertion. Predicates may be recursive; their meaning is defined as the least fixpoint of their definitions. This is well-defined since recursive occurrences must occur in positive positions. Another way to look at this is to see a predicate definition as an inductive definition with a single inference rule. For example, the following separation logic predicate describes a null-terminated linked list that stores the mathematical list of values $\alpha$ :

$$
\begin{aligned}
& \text { predicate } \operatorname{list}(\ell, \alpha) \equiv \\
& \quad \ell=0 \wedge \operatorname{emp} \wedge \alpha=\epsilon \\
& \vee \exists v, t, \alpha^{\prime} . \ell \mapsto v * \ell+1 \mapsto t * \operatorname{list}\left(t, \alpha^{\prime}\right) \wedge \alpha=v \cdot \alpha^{\prime}
\end{aligned}
$$

where $\cdot$ denotes prepending an element to a list.

Encoded into VeriFast syntax, this becomes:

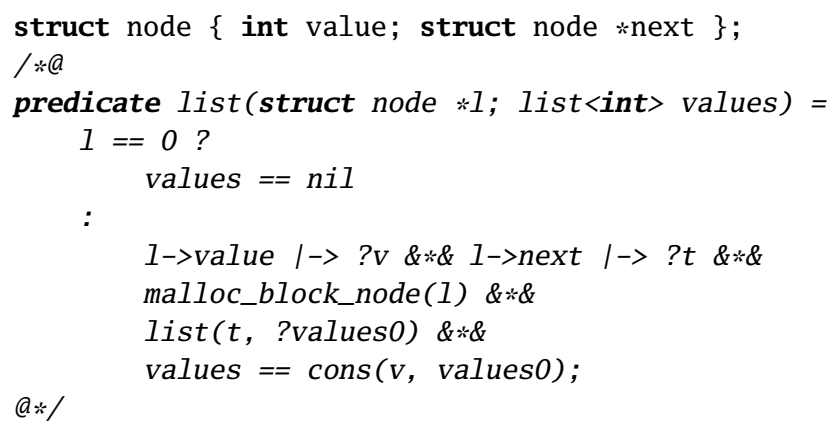

VeriFast does not support disjunctions at the level of separation logic assertions, because this would entail the need for backtracking in the symbolic execution engine (see below), which we do not do for the sake of performance, predictability, and diagnosability. (However, it does support disjunctions at the level of boolean expressions, i.e. assertions which do not mention separation logic constructs, because these are passed to the underlying SMT solver (see below).) Therefore, we use a conditional assertion (of the form cond ? asn1 : asn2) as the body of the predicate. We model the contents of the data structure using the mathematical inductive datatype list $<\mathrm{t}>$ defined as follows in the library that ships with VeriFast:

inductive list $<t>=\operatorname{nil} \mid \operatorname{cons}(t$, lis $t<t>)$;

Separating conjunction is written as $\& * \&$. Points-to assertions are written as $\mid->$. The built-in predicate malloc_block_node(1) denotes the permission to call free on the pointer 1 ; i.e. it encodes the fact that 1 was allocated using malloc (rather than being allocated on the stack, as a global variable, or as part of a larger object). VeriFast does not support general existential quantification; it only supports pattern matching: arguments of predicate assertions may be of the form ?x, in which case the assertion is implicitly existentially quantified over $\mathrm{x}$. This construct binds $\mathrm{x}$; its scope includes subsequent separating conjuncts. For example, the variable $t$ bound to the value of the next field of 1 in the assertion l->next $\mid->$ ?t above is used subsequently in list( $t$, ?values 0$)$. This restricted form of existential quantification enables a simple algorithm for checking assertions (see below).

VeriFast verifies each function against its contract through symbolic execution. This is similar to what an interpreter does, except that symbolic states are used instead of concrete states. A single symbolic state may represent many (even infinitely many) concrete states, thus allowing a finite number of symbolic executions to "cover" all potentially infinitely many concrete executions. In VeriFast, a symbolic state consists of a symbolic store, a symbolic heap, and a path condition. The symbolic store maps each local variable that is currently in scope to its symbolic value, which is a term of first-order logic that may contain symbols. The symbolic heap is a multiset of heap chunks of the form $\mathrm{p}\left(a_{1}, \ldots, a_{n}\right)$ where $\mathrm{p}$ is a separation logic predicate name (either built-in or user-defined) and $a_{1}, \ldots, a_{n}$, the chunk arguments, are terms of first-order logic. For a given interpretation of the logical symbols that appear in the chunk arguments, a given symbolic heap represents all concrete heaps that satisfy the separation logic assertion obtained by taking the separating conjunction of the heap chunks, interpreted as predicate assertions. The full set of concrete states represented by a symbolic state is obtained by taking the union of the sets of represented concrete states for all logical symbol interpretations that satisfy the path condition, which is a set of formulae of first-order logic.

The core operations of symbolic execution are assertion production and assertion consumption. Producing an assertion that is a boolean expression evaluates the expression under the symbolic store to yield a logical formula and adds this formula to the path condition. Producing a predicate assertion adds a corresponding chunk to the symbolic heap. Any existentially quantified chunk arguments are bound to fresh logical symbols. Producing a separating conjunction first produces the left-hand side, and then produces the right-hand side. Producing a conditional assertion forks the symbolic execution: on 
one branch, the condition is added to the path condition and the first branch's body is produced; on the other branch, the negation of the condition is added to the path condition and the second branch's body is produced.

The path condition is not actually maintained as a set of formulae; rather, during symbolic execution, VeriFast interacts with an SMT solver. The path condition corresponds to the state of the SMT solver. Adding a formula means "pushing" the formula into the SMT solver. If, after pushing a formula, the SMT solver reports that the resulting set of formulae is inconsistent, VeriFast aborts the current symbolic execution path, since it is infeasible, and continues with the next one. Aborting a symbolic execution path includes "popping" the state of the SMT solver to restore the state that existed at the most recent branch point.

Consuming an assertion that is a boolean expression evaluates the expression under the symbolic store to yield a formula and then asks the SMT solver to prove that formula from the path condition; if it fails, VeriFast reports an error. Consuming a predicate assertion looks for a matching chunk in the symbolic heap. If no match is found, an error is reported. Otherwise, existentially quantified variables in the predicate assertion are bound to the corresponding arguments of the matching chunk and the chunk is removed from the symbolic heap. Consuming a separating conjunction first consumes the left-hand side, and then the right-hand side. Consuming a conditional assertion proceeds analogously to production.

VeriFast verifies a function by first producing its precondition, then symbolically executing its body, and then consuming its postcondition. If any heap chunks are left in the symbolic heap after this last step, VeriFast reports that the function leaks heap chunks: this indicates a potential memory leak in the $\mathrm{C}$ program. Symbolic execution of an assignment to a struct field first consumes the heap chunk corresponding to the struct field, and then produces the same chunk with an updated value argument. When a struct $s$ is allocated using malloc, for each field $f$ of $s$ a chunk $s_{-} f\left(\ell, v_{f}\right)$ is produced, where $\ell$ is a fresh symbol denoting the address where the struct was allocated, and $v_{f}$ is a fresh symbol denoting the (unspecified) initial value of the field. Additionally, a malloc_block_s $(\ell)$ chunk is produced, denoting that the struct was allocated using malloc and therefore may be freed using free. The points-to notation $1->$ next $\mid->$ ?t in the example above is syntactic sugar for the predicate assertion node_next $(1, ? \mathrm{t})$.

Symbolic execution of function calls proceeds by first consuming the callee's precondition and then producing its postcondition. Notice that this approach implicitly applies separation logic's frame rule: any heap chunks that exist at the call site and that are not consumed by the precondition remain available to the caller in their original state. In our experience, this approach, involv- ing mostly just pattern matching, performs much better than verification condition generation-based approaches where the frame condition must be encoded into the input to the SMT solver using quantifiers that range over all memory locations, stating that all locations not declared as modified by the callee remain unchanged.

Sometimes, the symbolic heap must be rewritten into an equivalent form. For example, if the symbolic heap contains just the chunk list( 1 , values), and we wish to access the value field of the first node of 1 , we need to open the list chunk. This means replacing the chunk with its definition. The converse operation is closing a chunk, which consumes the chunk's definition and produces the chunk itself. In general, VeriFast does not open or close chunks automatically, again to preserve predictable and diagnosable performance. Rather, the user must insert open and close ghost commands into the code. However, if a predicate is marked precise, by inserting a semicolon between its input parameters and its output parameters, VeriFast will attempt to automatically open and close it when appropriate, provided the values of the input arguments are known. A predicate is precise if, for a given valuation of the input arguments and for a given heap, there is at most one subheap and at most one valuation of the output arguments that satisfy the predicate.

For modeling and reasoning about data, such as the contents of the linked list in the example above, the VeriFast approach is oriented towards inductive datatypes and primitive recursive functions (called fixpoint functions) over these. An example of the former is the list $<t>$ datatype, and an example of the latter is the length function, defined as follows in the library that ships with VeriFast:

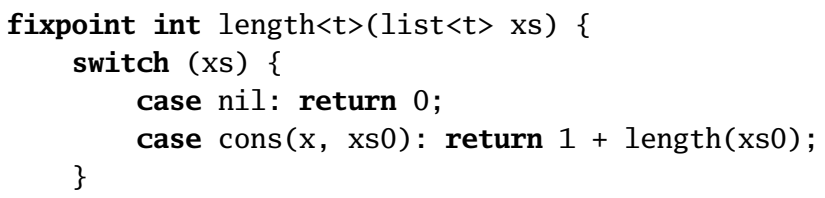

Like in proof assistants that support primitive recursive functions such as Isabelle/HOL [9] and Coq [1], a fixpoint function's body must be a case split over one of its arguments (called its inductive argument), and each recursive call must structurally decrease this argument; i.e. the callee's value for this argument must be a component of the caller's. In the example, the recursive call length(xs0) is allowed because xs0 is a component of xs. Inductive datatypes and fixpoint functions have a simple and clear mathematical meaning; they are expressive enough for most purposes; and we can offer welldefined and predictable automated reasoning for them: in principle, only reduction occurs automatically. Reduction equates a fixpoint function call whose inductive argument is a constructor with the body of the corresponding case in the function's definition. VeriFast is purposely incomplete: it does not automatically perform case splits 
or induction. The goal is to preserve predictable performance. The reasoning is implemented by encoding the inductive datatypes and fixpoint functions into the background theory of the SMT solver using uninterpreted function symbols and axioms for injectiveness and disjointness (but not exhaustiveness) of constructors and for reduction of fixpoint functions.

While no automatic induction occurs for reasoning about inductive datatypes or separation logic predicates, manual induction is supported by writing lemma functions: a lemma function is like a regular $\mathrm{C}$ function, except that it is defined within an annotation and VeriFast checks that it has no side-effects and that it terminates. VeriFast supports a number of termination arguments; the most important ones are structural induction on an inductive argument and induction on the derivation of a separation logic predicate. The latter is based on the fact that each non-built-in chunk was produced from built-in chunks by a finite number of close operations. For example, an inductive proof of the fact that the length of a list is always nonnegative can be given in VeriFast as follows:

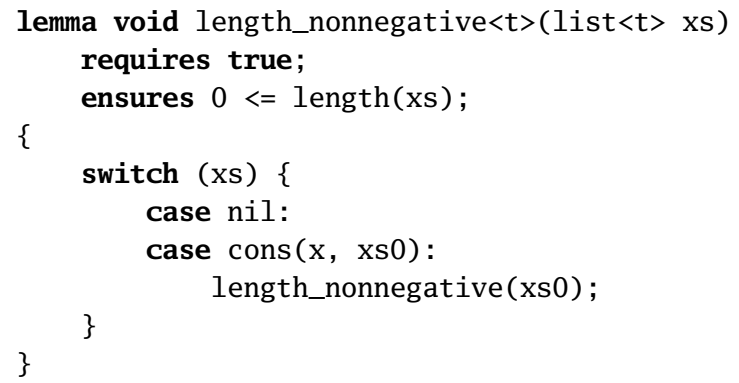

Then, to obtain this fact for a given specific inductive list value, one can either call this lemma explicitly as a ghost command, or one can declare the lemma as a lemma_auto, in which case the lemma is passed as an axiom to the SMT solver and applied automatically. This introduces a risk of degraded performance or nontermination of the SMT solver; we leave this responsibility to the user. Note: only autolemmas whose contract does not involve separation logic constructions are passed to the SMT solver; others are applied by the symbolic execution engine during chunk production and consumption.

As will be illustrated by the challenge solutions below, the VeriFast approach for verifying properties of an imperative program often comes down to using separation logic to establish a correspondence between the imperative program and a pure functional version of it, and then separately verifying the desired properties with respect to the pure functional program, the latter in ways very similar to how one develops theories in proof assistants like Coq and Isabelle/HOL. This stands in some contrast with approaches that are oriented less towards inductive datatypes and fixpoint functions and more towards quantifier-rich specifications, which tend to lead to more direct statements and proofs of the desired properties, but for which it might be harder to offer a pre- dictable, diagnosable, well-performing proof authoring experience. We believe no one particular approach currently being proposed in the verification community is a clear winner, and each has important benefits and contributions, and as the community grows to understand better the various trade-offs involved we will learn how to combine the various approaches to best tackle particular types of problems.

\section{Challenge 1: Longest Common Prefix}

\subsection{Challenge}

Essentially, the challenge was to specify and verify the functional correctness of the following $\mathrm{C}$ function, which, given an array of integers a of length $\mathrm{N}$, returns the length of the longest common prefix of the subarrays starting at indices $\mathrm{x}$ and $\mathrm{y}$.

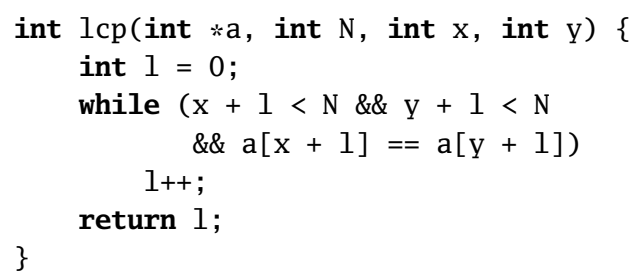

(We did not, either during or after the competition, attempt to solve the part marked Advanced of the challenge, which was to verify a program that computes the longest repeated substring of a given string.)

\subsection{Competition Solution 1}

The first solution that we came up with during the competition is shown in Figure $1 .^{1}$

The solution consists of the $\mathrm{C}$ function definition given in the challenge (transformed slightly; see below), augmented with VeriFast annotations. These take the form of $\mathrm{C}$ comments marked with an @ sign.

The specification of the $\mathrm{C}$ function consists of a precondition, given by the requires clause, and a postcondition, given by the ensures clause. The precondition states that the function must receive read permission to elements 0 , inclusive, through $\mathrm{N}$, exclusive, of the array $\mathrm{a}$, and that arguments $\mathrm{x}$ and $\mathrm{y}$ are within appropriate bounds. In VeriFast, a function's precondition must require access permissions for all memory locations that the function accesses, except for the ones that it allocates itself or for which it obtains permission through some other means (such as acquiring a mutual exclusion lock). In particular, to write to a memory location,

1 The only differences with the actual submitted code are 1) that this version uses an improved notation for array permissions which was introduced in the December 2012 release of VeriFast, and 2) that we removed some ghost statements that turned out to be superfluous. 


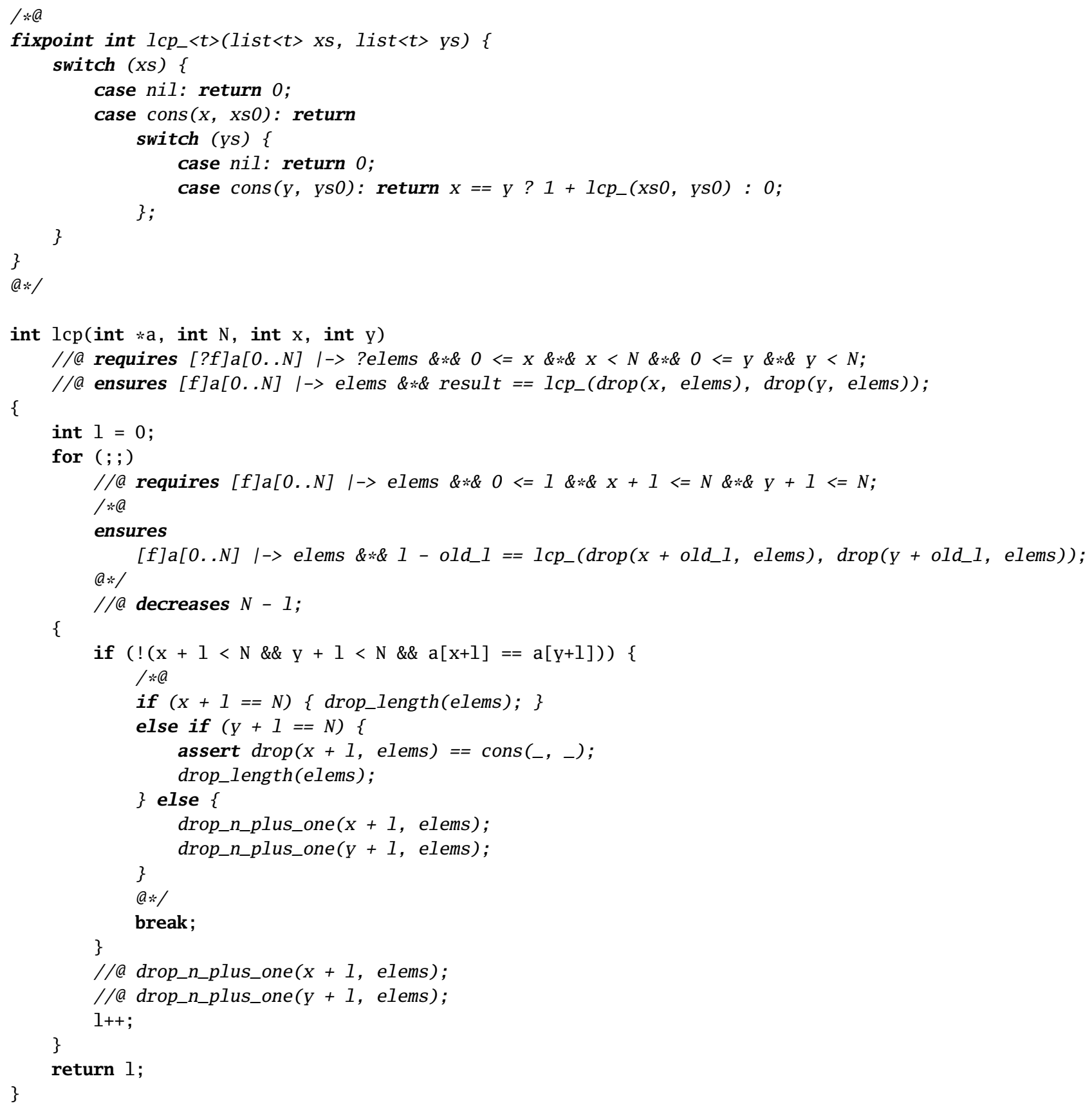

Fig. 1. Our first competition solution to Challenge 1

full permission is required; to only read from a memory location, some fractional permission [2] is sufficient. A full permission can be split into a number of fractional permissions and later reassembled into a full permission. Each fractional permission has a coefficient, a rational number between 0 , exclusive, and 1, inclusive. (A coefficient of 1 denotes a full permission.) The coefficient, if not 1 , is written inside a pair of brackets preceding the permission. The notation [?f] denotes that an arbitrary fraction is sufficient, and binds the actual coefficient to ghost variable $\mathrm{f}$.
The precondition also binds the contents of the specified array elements, represented as a value of the ghost type list<int>, to the ghost variable elems. The ghost variables bound in the precondition are in scope in the postcondition. This allows the postcondition to specify that the function returns the same fractional permission $f$ that it received, and that the return value of the function is the longest common prefix of the sublists of elems obtained by dropping the first $\mathrm{x}$ and $\mathrm{y}$ elements, respectively. The longest common prefix of two lists is specified using the fixpoint function lcp_ defined recursively in the 
annotation preceding the $\mathrm{C}$ function. This function uses an extended switch notation to perform a case analysis on the list values passed in as arguments. Type list is defined inductively in the built-in VeriFast header file list.h as follows:

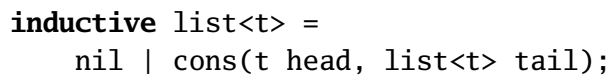

A list of elements of type $t$ is either empty (denoted by constructor nil) or non-empty, with a first element (called the head) of type $t$ and a remainder (called the tail) of type list<t>. As another example of a fixpoint function, the function drop used in the postcondition is defined in list.h as follows:

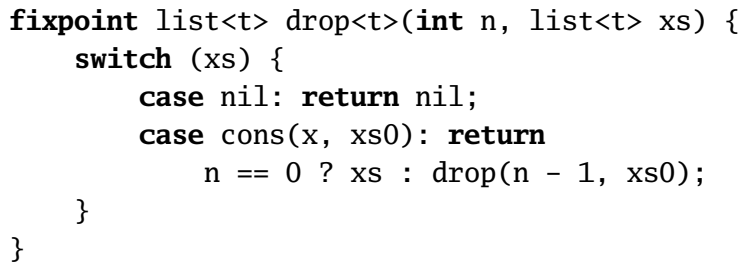

Since the list type that we use to model the contents of the array is defined inductively, and the functions $\mathbf{l c p}_{-}$and drop used in the specification are defined recursively, a recursive implementation of the $\mathrm{C}$ function would have been closer to the specification and therefore easier to verify. However, the implementation given in the challenge is iterative. Applying the classical loop verification proof rule here, with a loop invariant, would be somewhat painful. However, an alternative proof rule for loops was proposed recently [14] that allows a loop to be verified as if it was a tail-recursive function. We have implemented this proof rule in VeriFast; specifically, for annotating a loop, the user can choose to provide a loop contract instead of a loop invariant. For example, the loop

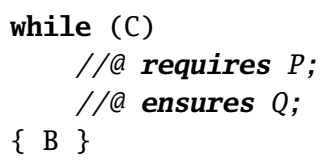

is verified by VeriFast as if it was a call of a local function:

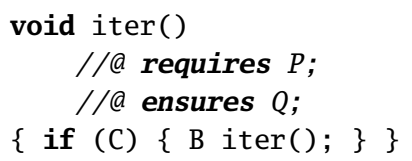

We have applied this feature in the solution. However, applying this feature means that VeriFast checks that the loop postcondition holds when the loop ends (i.e., when $C$ is false). This means that the postcondition must follow from the conjunction of the precondition and the negation of the loop condition. In general, however, VeriFast cannot prove this implication without help. Helping VeriFast means inserting extra ghost statement annotations, such as lemma applications and case splits. But in the case of a regular while loop, there is no way for the user to insert ghost code between the start of the loop iteration and the exit from the loop. For our solution, to work around this problem we rewrote the given function body slightly: we moved the condition check into the body of the loop using an if statement and a break statement; this gave us the opportunity to insert ghost statements between the point where the negation of the loop condition is established and the point where the loop is exited (and where VeriFast checks that the postcondition holds). Schematically, we rewrote our loop into the form

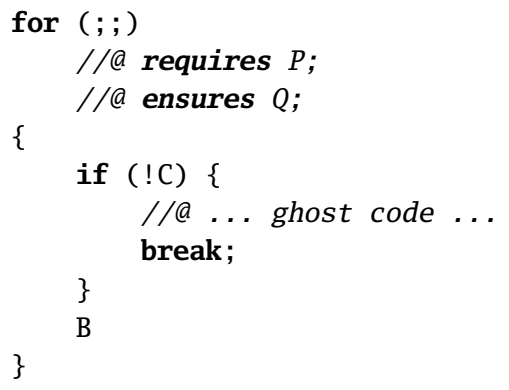

which is verified as if it was of the form

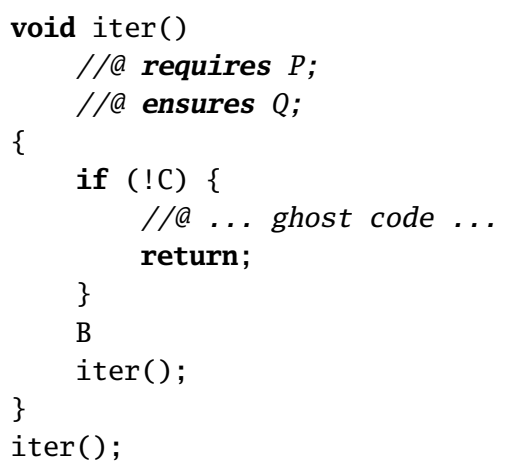

The loop contract in our solution is similar to the function contract, except that the postcondition states that the loop "returns" the length of the longest common prefix of the subarrays starting at $\mathrm{x}+$ old_l and $\mathrm{y}+$ old_l, where old_l denotes the value of variable 1 at the start of the current loop "call" (i.e. the imaginary call of the tail-recursive function). The "return value" is embodied in the difference between the new value of 1 and the old value of 1 . Notice that this contract is indeed appropriate: a) it is established by the base case, i.e. when the loop exits; b) given that the contract holds for a "tail-recursive call" of the loop, it holds for the "caller"; c) the loop contract implies the function contract, considering that the initial value of 1 is 0 and that the function returns the final value of 1 .

Proving the cases (a) and (b) required a number of ghost statements. For case (a), the case distinction that happens in the loop condition had to be repeated in the annotations, and for each case, additional ghost code was necessary to establish the loop postcondition. Specifically, if $x+1==\mathrm{N}$, the postcondition follows from the fact that $\operatorname{drop}(x+1$, elems $)$ equals nil, but the latter fact is not immediately obvious to VeriFast since it re- 
quires an inductive proof, which VeriFast does not attempt to construct. The solution is to call a lemma function that provides this fact in its postcondition. Such a lemma function, called drop_length, is declared in header file list.h as follows:

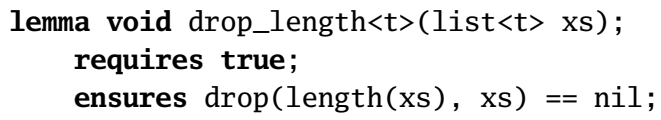

The second case of the loop condition, where $\mathrm{x}+$ $1 !=\mathrm{N}$ but $\mathrm{y}+1==\mathrm{N}$, can be dealt with similarly, by another call of drop_length to show that $\operatorname{drop}(\mathrm{y}+1$, elems) equals nil, except that we also need to perform a case split on $\operatorname{drop}(x+L, N)$, since otherwise VeriFast will not reduce the first switch expression in the body of $1 \mathrm{cp}_{-}$. (VeriFast never performs case splits implicitly, since this can easily lead to performance degradation and even non-termination of the verifier.) This case split is provoked by asserting that $\operatorname{drop}(x+1$, elems $)$ matches the pattern cons $\left(\_, \ldots\right)$. Such a pattern-matching assertion is checked by doing a case split and checking that all non-matching cases lead to absurdity.

The final subcase of case (a) is the case where the corresponding array elements are unequal. From this fact, which is expressed in terms of the elems list as $n t h(x+1$, elems $) !=n t h(y+1$, elems $)$, VeriFast must derive that the $1 c p_{-}$function goes into the last branch of its body. For this to happen, VeriFast must see that $\operatorname{drop}(\mathrm{x}+1$, elems) and $\operatorname{drop}(y+1$, elems) are nonempty (i.e. they match cons $\left.\left(_{-},{ }_{-}\right)\right)$and that their first elements are $n t h(x$ +1 , elems $)$ and $n t h(y+1$, elems $)$. This is what the calls of the drop_n_plus_one lemma provide, which is declared in list.h as follows:

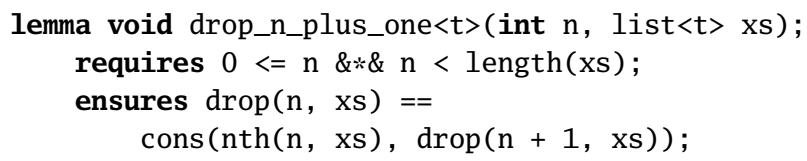

For the case (b) where the loop does not end, i.e. the recursive case, we need to prove that the call of $1 \mathrm{cp}_{-}$ also hits its recursive case. Similarly to the last subcase of (a), this is accomplished with two calls of lemma drop_n_plus_one.

\subsection{Competition Solution 2}

The second solution we came up with during the competition is shown in Figure 2. ${ }^{2}$

As in our first solution, the precondition of 1cp demands read permission to the first $\mathrm{N}$ elements of $\mathrm{a}$, and requires that $\mathrm{x}$ and $\mathrm{y}$ lie within proper bounds. The postcondition returns the requested permissions to the caller. However, the relation between the function's return value and the elements of the array is described in

\footnotetext{
2 The only differences with the actual submitted code are 1) that this version is written in C instead of Java, 2) that it uses the improved notation for array permissions as before, and 3) that quantifiers are expressed via forall instead of forall_nth.
}

a more declarative style. More specifically, the postcondition states 1 ) that the return value lies within proper bounds, 2) that the subarrays of a starting at $\mathrm{x}$ and $\mathrm{y}$ share a common prefix of length result, and 3) that no longer common prefix exists. In the first solution, these properties can be derived from the definition of the func-

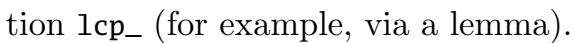

The second property of result is expressed via a universal quantifier: for all integers $i$ in the range 0 (inclusive) to result (exclusive), the element at index $x+i$ is equal to the one at $y+i$. Interestingly, this form of bounded quantification is not built into VeriFast. Instead, the specification library quantifiers.gh partially shown in Figure 3 declares the higher-order function forall. This function takes a list vs and a property $p$ (of type fixpoint( $t$, bool), which is VeriFast notation for the function type $t \rightarrow$ bool of functions that take a value of type $t$ as an argument and return a value of type bool) as arguments, and returns whether each element of vs satisfies p. In addition to the function itself, the library provides two lemmas, get_not_forall_witness and apply_forall, that can be used by clients to reason about forall. The former lemma returns an element of vs that does not satisfy p, provided forall(vs, p) does not hold. The latter lemma states that each member of the list satisfies $\mathrm{p}$ if forall(vs, p) holds.

One might reasonably try to express the fact that the sublists of list elems at offsets $\mathrm{x}$ and $\mathrm{y}$ have a common prefix of length result through an expression like

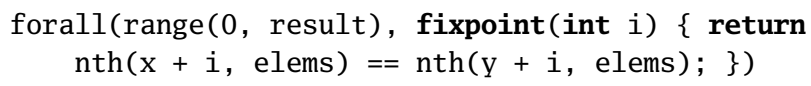

where fixpoint $(\mathrm{T} x)$ \{return $\mathrm{E}$; $\}$ would denote an anonymous fixpoint function that maps arguments $\mathrm{x}$ to results E. However, VeriFast currently does not support anonymous fixpoint functions. Therefore, the quantifier in the specification of $1 \mathrm{cp}$ uses the fixpoint sublists_equal_at_offset to state the desired property. This function is partially applied to elems, $\mathrm{x}$ and $\mathrm{y}$ in order to obtain a function from integers to booleans (as required by forall).

A disadvantage of providing quantification as a library is that VeriFast is often unable to automatically discharge proof obligations involving quantifiers. In such cases, the tool relies on the user to complete the proof (by inserting ghost code, which typically calls lemmas). For example, consider the body of the loop in Figure 2. The ghost statements in the body are crucial for VeriFast to be able to deduce that the loop preserves the loop invariant. In particular, they prove that the quantifier holds for range $(0,1)$. They do so by contradiction: assume that the quantifier does not hold, and derive absurdity. The absurdity proof in the solution first obtains the index of an element for which the quantifier does not hold, and then proceeds by case analysis on whether the element is the last one or not. If not, absurdity follows from the fact that the quantifier holds for range $(0,1-1)$; oth- 


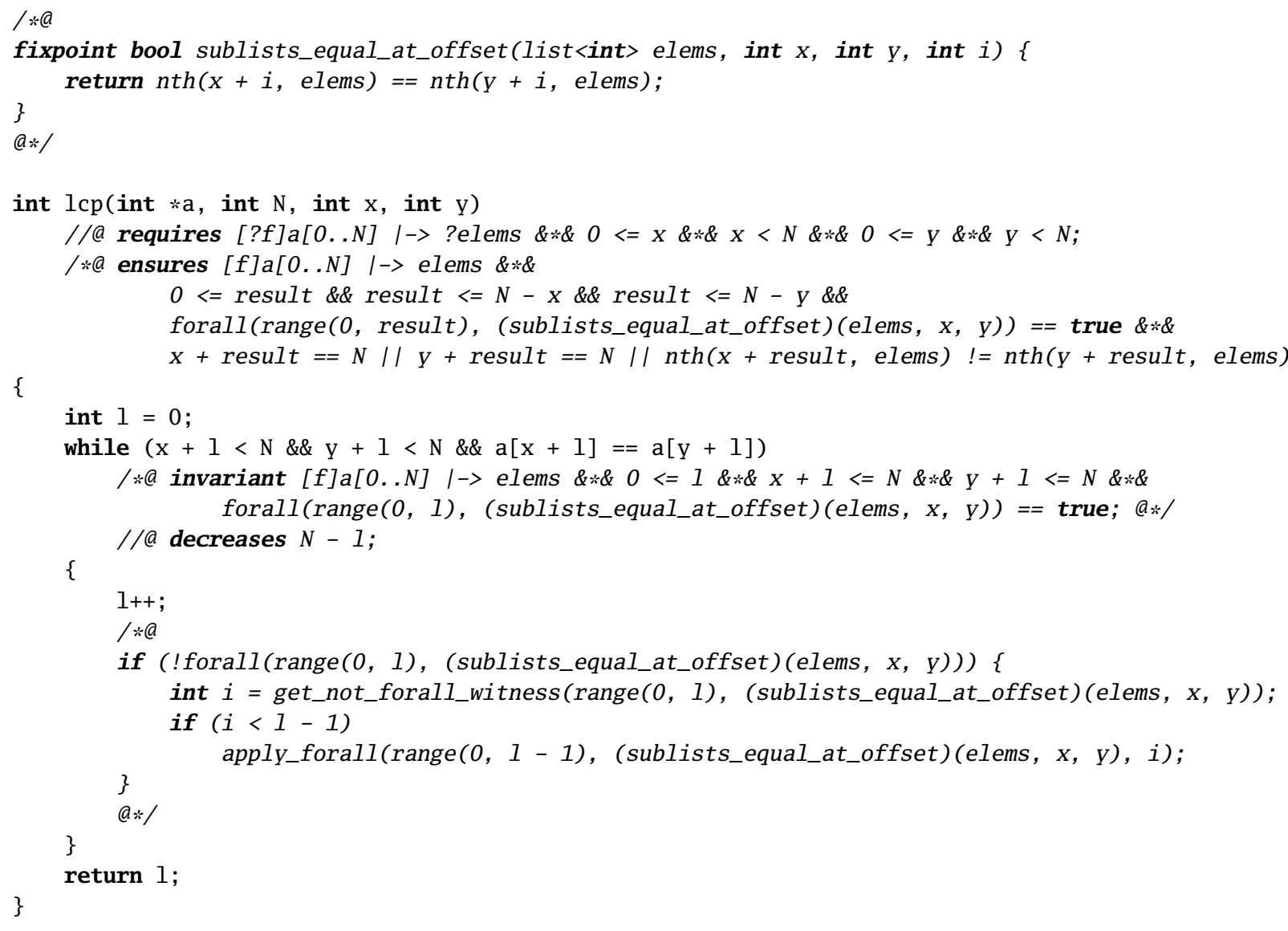

Fig. 2. Our second competition solution to Challenge 1

erwise, it follows from the fact that the loop condition holds.

VeriFast also supports native quantifiers, in the form of forall_(int i; E) expressions. By native, we mean that these quantifiers are encoded as quantifiers in the underlying SMT solver (instead of as applications of the uninterpreted function symbol forall). After the competition, we also solved the first challenge making use of such native quantifiers. In particular, the call of forall in the loop invariant in Figure 2 is replaced in this solution by:

forall_(int $i ; i<0|| 1<=i||$

sublists_equal_at_offset(elems, x, y, i))

and similarly in the postcondition. The key advantage of native quantifiers is that the SMT solver can automatically discharge many proof obligations involving such quantifiers, and therefore that the developer needs to provide fewer annotations to help the tool complete the proof. For example, the ghost statements in the body of the loop in Figure 2 can be omitted when using native quantifiers. However, in our experience relying on native quantifiers also has a number of disadvantages. In particular, the time needed by the SMT solver to discharge proof obligations involving general quantifiers (and even whether it can discharge the proof obligation at all) can be extremely sensitive to minor changes to the program and the specifications. As a consequence, it can be hard to diagnose (and remedy) the exact cause of a verification failure. For example, when we tried to make use of native quantifiers to solve the first challenge during the competition (the postcondition was expressed directly in terms of function nth, without using auxiliary function sublists_equal_at_offset), verification failed; later diagnosis indicated that the SMT solver's heuristics for instantiating quantifiers failed to produce the required instantiations. Rewriting the quantifier using function sublists_equal_at_offset was sufficient to make the proof go through.

\section{Challenge 2: Prefix Sum}

\subsection{Challenge}

The challenge was essentially to specify and verify functional correctness of an algorithm for computing the prefix sums of a given array of integers, expressed in Java in Figure 4: When method prefixSums returns, the element of array a at index $i$ should contain the sum of the initial 


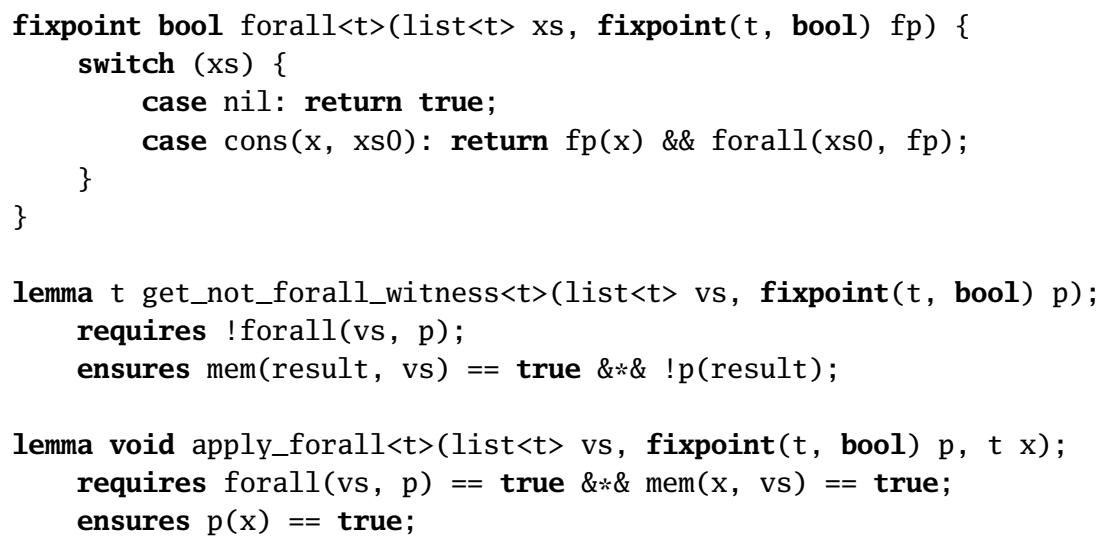

Fig. 3. Part of quantifiers.gh, a specification library for quantification. Fixpoint function mem(x, xs) checks if list xs contains element $\mathrm{X}$.

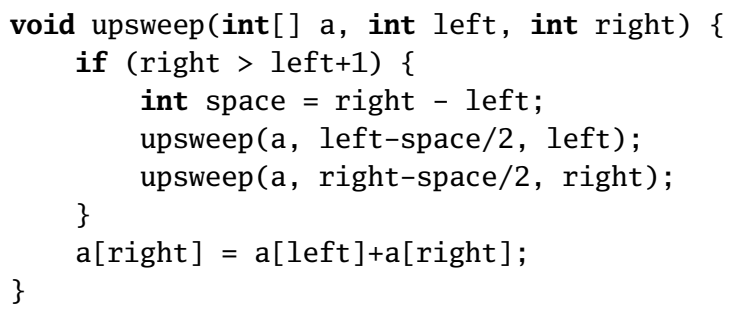

void downsweep(int[] a, int left, int right) \{ int $\mathrm{tmp}=\mathrm{a}$ [right];

Fig. 4. Prefix sum algorithm of Challenge 2

values of elements 0 through $i-1$, for all $i$, provided that the length of the array is a power of two greater than 1 .

\subsection{Competition Solution}

A cleaned version of the solution we submitted during the competition is shown in Figures 5 and 6.

Our specification for method prefixsums requires in its precondition that the array can be described using the separation logic predicate tree $0 . \operatorname{tree} 0(a, l, r, t)$ says that $l<r$ and array $a$ stores the leaves of the complete binary tree $t$ in elements $s$ through $r$ with $s=r+1-$ $2(r-l)$. That is, $l$ is the index of the element storing the rightmost leaf of the left subtree of $t$ and $r$ is the index of the element storing the rightmost leaf of the right subtree of $t$. For the precondition of method prefixsums, this means that the length of array a must be a power of two greater than 1, and its contents are bound to ghost variable values in the form of a value of type tree that is complete (i.e. all leaves are at the same depth). Type tree is defined at the top of our solution.

The postcondition states that when the method returns, the array contents are described by the fixpoint function prefix_sums applied to values, with initial value 0 of the accumulator. This function, defined earlier in the solution, returns the list of prefix sums of the values stored in the given binary tree, incremented by the given accumulator value.

To explain the proof of method prefixSums, we first explain the specification and proof of methods upsweep and downsweep, and of lemma function tree2_prefix_sums.

The specification of method upsweep requires that when the method is called, the array must be described by predicate tree0, as described above. It ensures that when the method returns, the array is described by predicate treel with the same arguments, except that the rightmost element is separately specified to contain the sum of the initial values of the relevant elements of the array. $\operatorname{tree} 1(a, l, r, t)$ states that $l<r$, that $t$ is a complete binary tree with $2(r-l)$ leaves, and that the array element at index $s+i-1$ contains the sum of the leaves of the largest subtree of $t$ for which $t$ 's $i$ 'th leaf is the rightmost leaf, where $s=r+1-2(r-l)$ as before, for all $1 \leq i<2(r-l)$. The rightmost element (the element at index $r$ ) is not described by the tree1 predicate; this prevents the predicate from encapsulating the permission required for the array element assignments in upsweep and prefixSums. 


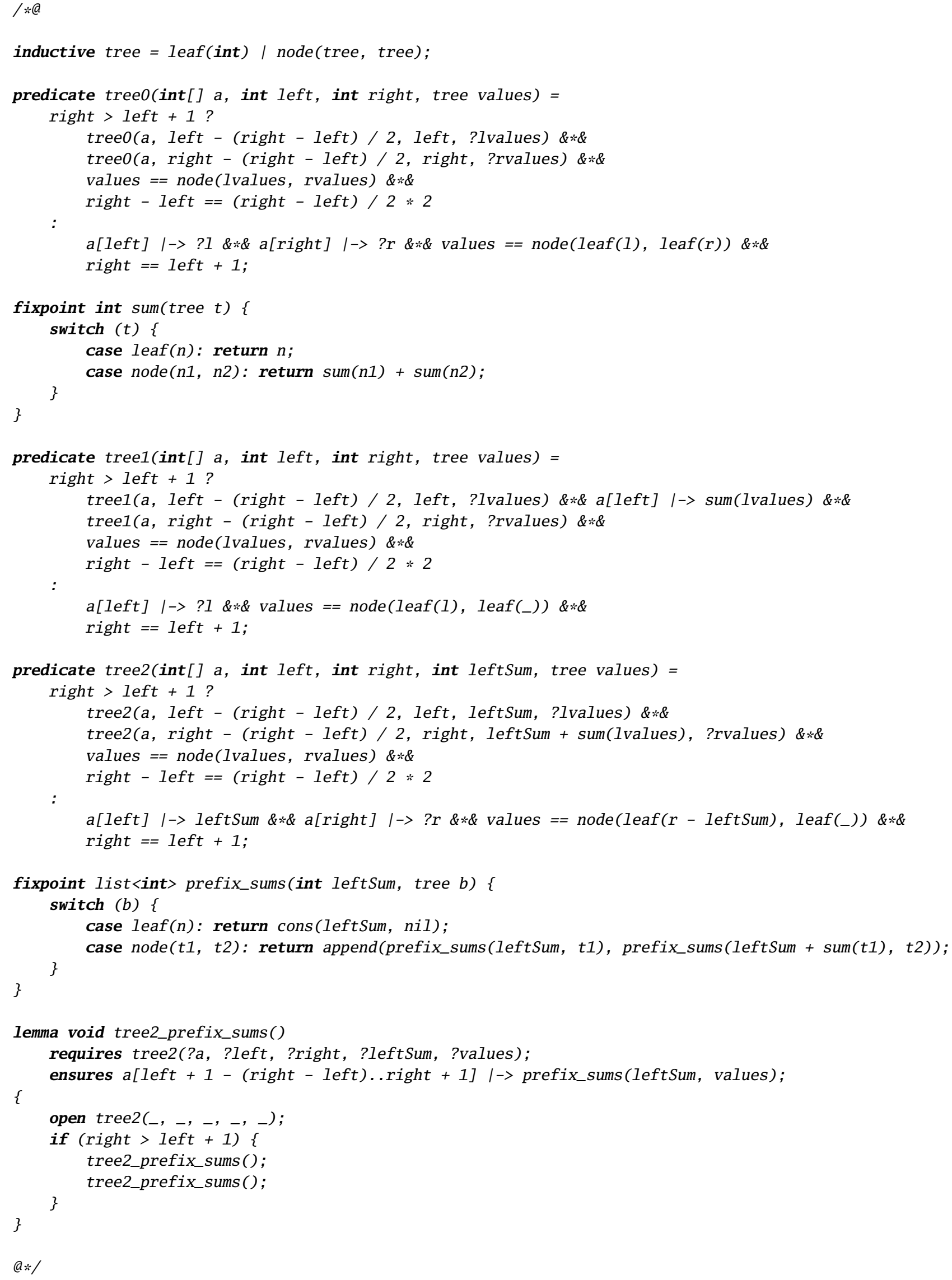


class PrefixSumRec \{

public static void upsweep(int[] a, int left, int right)

//@ requires tree0(a, left, right, ?values);

//@ ensures tree1(a, left, right, values) \&*\& a[right] /-> sum(values);

\{

//@ open $\operatorname{treeO}\left({ }_{-}, \ldots,-,-\right)$;

if (right $>$ left +1 ) \{

int space $=$ right - left;

upsweep (a, left-space/2, left);

\}

upsweep (a,right-space/2,right);

$\mathrm{a}[$ right $]=\mathrm{a}[$ left $]+\mathrm{a}[$ right $]$;

//@ close treel(a, left, right, values);

\}

public static void downsweep (int[] a, int left, int right)

//@ requires tree1(a, left, right, ?values) \&*\& a[right] /-> ?leftSum;

//@ ensures tree2(a, left, right, leftSum, values);

\{

//@ open $\operatorname{tree1}\left({ }_{-}, \ldots, \ldots, \ldots\right)$;

int tmp $=\mathrm{a}[$ right $]$;

$\mathrm{a}[$ right $]=\mathrm{a}[$ right $]+\mathrm{a}[$ left $]$

$\mathrm{a}[$ left $]=\mathrm{tmp}$;

if (right $>$ left +1 ) \{

int space $=$ right - left;

downsweep (a, left-space/2, left);

\}

downsweep (a,right-space $/ 2$, right) ;

\}

//@ close tree2(a, left, right, leftSum, values);

public static void prefixSums(int [] a)

//@ requires a $!=$ null \&*\& tree0(a, a.length / 2 - 1, a.length - 1, ?values);

//@ ensures $a[\ldots] /->\operatorname{prefix\_ sums(0,values);}$

\{

upsweep(a, a.length / $2-1$, a.length -1 );

$\mathrm{a}[\mathrm{a}$. length -1$]=0$;

downsweep(a, a.length / 2 - 1, a.length - 1);

//@ tree2_prefix_sums();

\}

public static void main(String[] args)

//@ requires true;

//@ ensures true;

\{

int [] $\mathrm{a}=\{3,1,7,0,4,1,6,3\}$;

//@ close $\operatorname{treeO}(a, 0,1, \ldots)$;

//@ close $\operatorname{treeO}(a, 2,3, \ldots)$;

//@ close $\operatorname{treeO}(a, 1,3, \ldots)$;

//@ close $\operatorname{treeO}(a, 4,5, \ldots)$;

//@ close $\operatorname{tree} 0(a, 6,7, \ldots)$;

//@ close $\operatorname{tree} 0(a, 5,7, \ldots)$;

//@ close $\operatorname{tree} 0(a, 3,7, \ldots)$;

prefixSums (a);

//@ assert $a[\ldots] /->\{0,3,4,11,11,15,16,22\}$;

\}

Fig. 6. Our competition solution to Challenge 2 (Part 2 of 2) 
For the verification of the body of upsweep, all that was required was an explicit unfolding of predicate tree0 and an explicit folding of predicate tree1. This is because the definition of the predicates matches closely the structure of the code.

The specification of downsweep requires in its precondition the state as it is left by upsweep, except that the value of a[right] is not constrained, and ensures that when the method returns, the relevant part of a is described by the predicate tree2. tree2 $(a, l, r, p, t)$ states that $l<r$, that $t$ is a complete binary tree with $2(r-l)$ leaves, and that the element of array $a$ at index $s+i-1$ contains the sum of $p$ and the first through $i-1$ 'th leaf of $t$, where $s=r+1-2(r-l)$ as before, for all $1 \leq i \leq 2(r-l)$.

Similarly to the proof of upsweep, the proof of the function downsweep only requires an explicit unfolding of predicate tree1 and an explicit folding of predicate tree2, again because the predicate definitions have been chosen to coincide with the structure of the code.

Finally, the lemma tree2_prefix_sums is used to show that predicate tree2 does indeed imply the prefix sums property. The proof is by straightforward induction on the derivation of the tree 2 predicate.

\section{Challenge 3: Iterative Deletion in a Binary Search Tree}

\subsection{The Challenge}

The challenge was to specify and verify functional correctness of the following algorithm that removes the minimum element from a binary search tree:

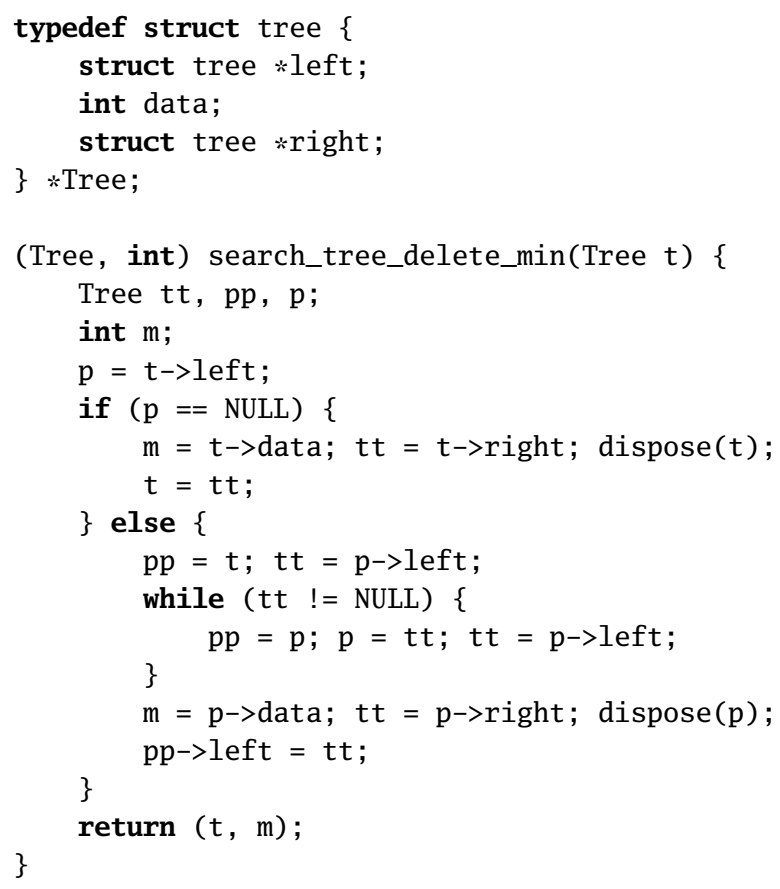

\subsection{Competition Solution}

The most challenging part of this task is the specification of the loop: the loop "returns" three pointers that point deep inside a tree data structure, and then the code after the loop modifies the tree through these pointers. The information provided by the loop specification should be sufficient to prove that the piece of code after the loop is safe, establishes the correct function result, and maintains the well-formedness of the binary search tree.

A cleaned-up version of the solution that we submitted during the competition is shown in Figures 7-8.

Before we describe how we addressed the loop specification problem, we first explain how we specified the function.

The function's precondition uses separation logic predicate Tree (not to be confused with program type Tree) to specify that pointer $t$ must point to a well-formed tree data structure, and to bind its contents to ghost variable vs. Furthermore, it requires that the tree be non-empty, and that it receive permission to write to the pointers $r 1$ and r2. The postcondition states that in the post-state r1 points to some pointer tresult which itself points to a well-formed tree data structure whose contents can be obtained by deleting the leftmost leaf of vs; furthermore, r2 points to the value of this leaf.

Notice that the data structure contents are specified as a value of inductive datatype tree, which specifies a precise tree shape, including the address of each node. While this does not break information hiding (since the predicate Tree need not comply with the shape information specified by the tree value; indeed, it could ignore all information except for the set of data values contained in the nodes), this is not a particularly clean choice. However, given the time constraints, we chose a datatype that would facilitate the proof of the body of the function.

The Tree predicate chosen for this particular proof does in fact follow the shape of the given tree value.

As for the proof of the body of the function, the case where the root of the tree is the leftmost node is trivial. We concentrate on the other case.

To address the abovementioned loop specification problem, for this solution we chose to have the loop return a "magic wand". A magic wand or separating implication $P * Q$ is a separation logic permission that means as much as " $Q$ except for $P$ ", i.e. one can derive $Q$ given both $P$ and $P \rightarrow Q$ :

$$
P *(P \rightarrow Q) \Rightarrow Q
$$

Our loop returns a magic wand that says: if you give me the left field of pp updated with the correct new value, then I will give you the tree data structure, well-formed, in its new state where the minimum element has been deleted. Indeed, when the loop ends, the tree is in the desired state, except that the left field of pp must still be updated to point to the right-hand child of $\mathrm{p}$. 


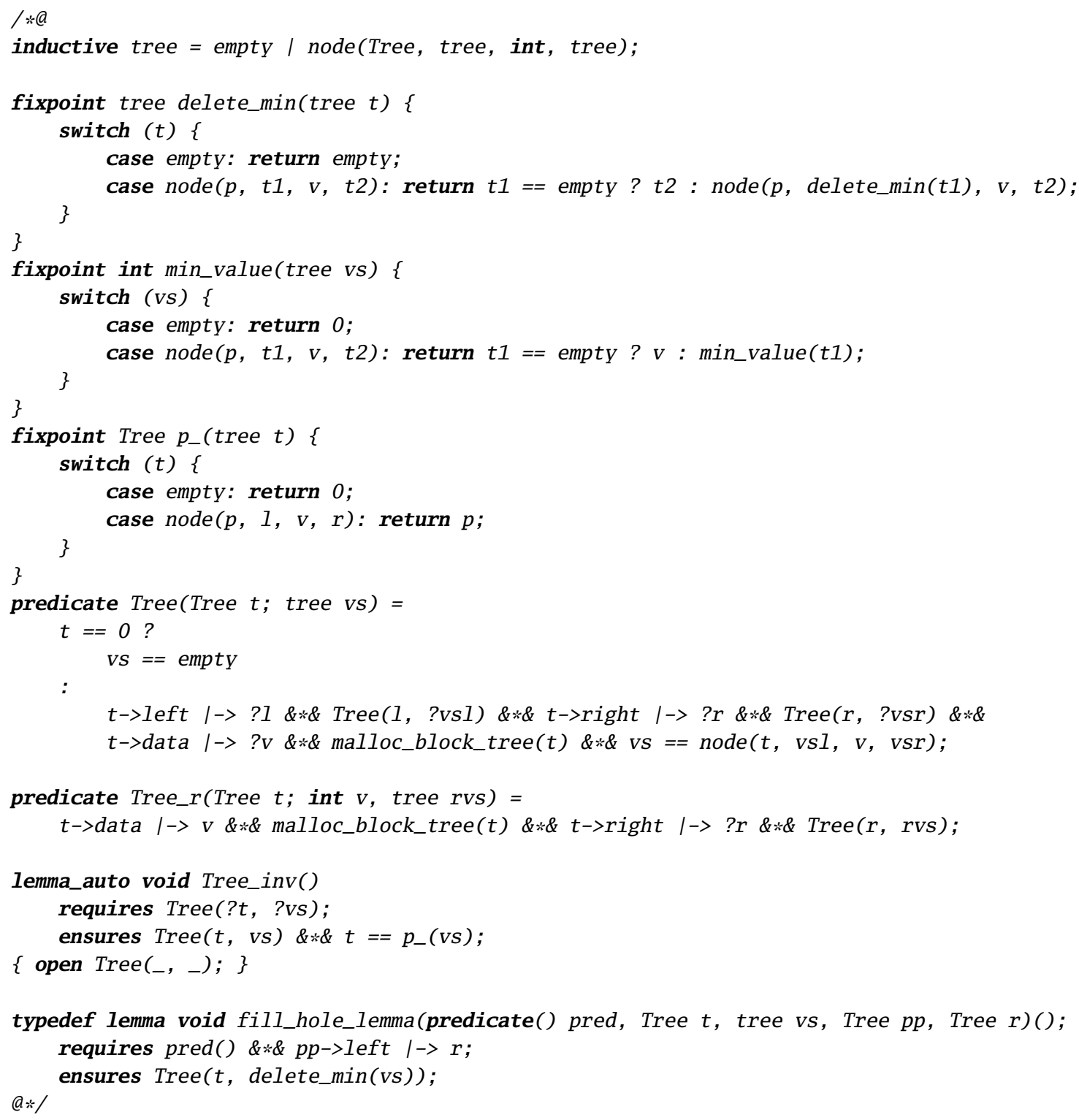

Fig. 7. Our competition solution for Challenge 3 (Part 1 of 2)

VeriFast does not have built-in support for magic wands. However, it supports lemma function pointers and parameterized lemma function types. Together, these features allow us to encode magic wands into VeriFast.

Specifically, VeriFast supports lemma function type definitions of the following form:

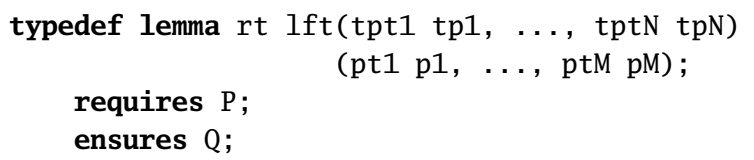

This defines a lemma function type lft with return type rt, lemma function type parameters tp1 through tpN, lemma function parameters $\mathrm{p} 1$ through $\mathrm{pM}$, precondition $\mathrm{P}$, and postcondition Q. Both the lemma function type parameters and the lemma function parameters may ap- pear in the precondition and the postcondition. Given this lemma function type definition, we can assert that some lemma function if complies with the type, instantiated with particular values v1 through vN for the lemma function type parameters, using a ghost command of the form

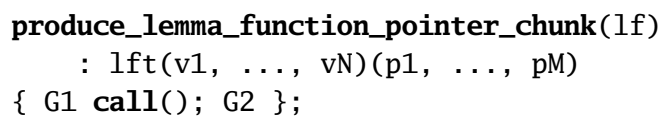

where G1 and G2 are optional sequences of ghost commands (such as open or close commands or lemma function calls) to aid in deriving the lemma function precondition from the lemma function type precondition and the lemma function type postcondition from the lemma function postcondition, respectively. This ghost 


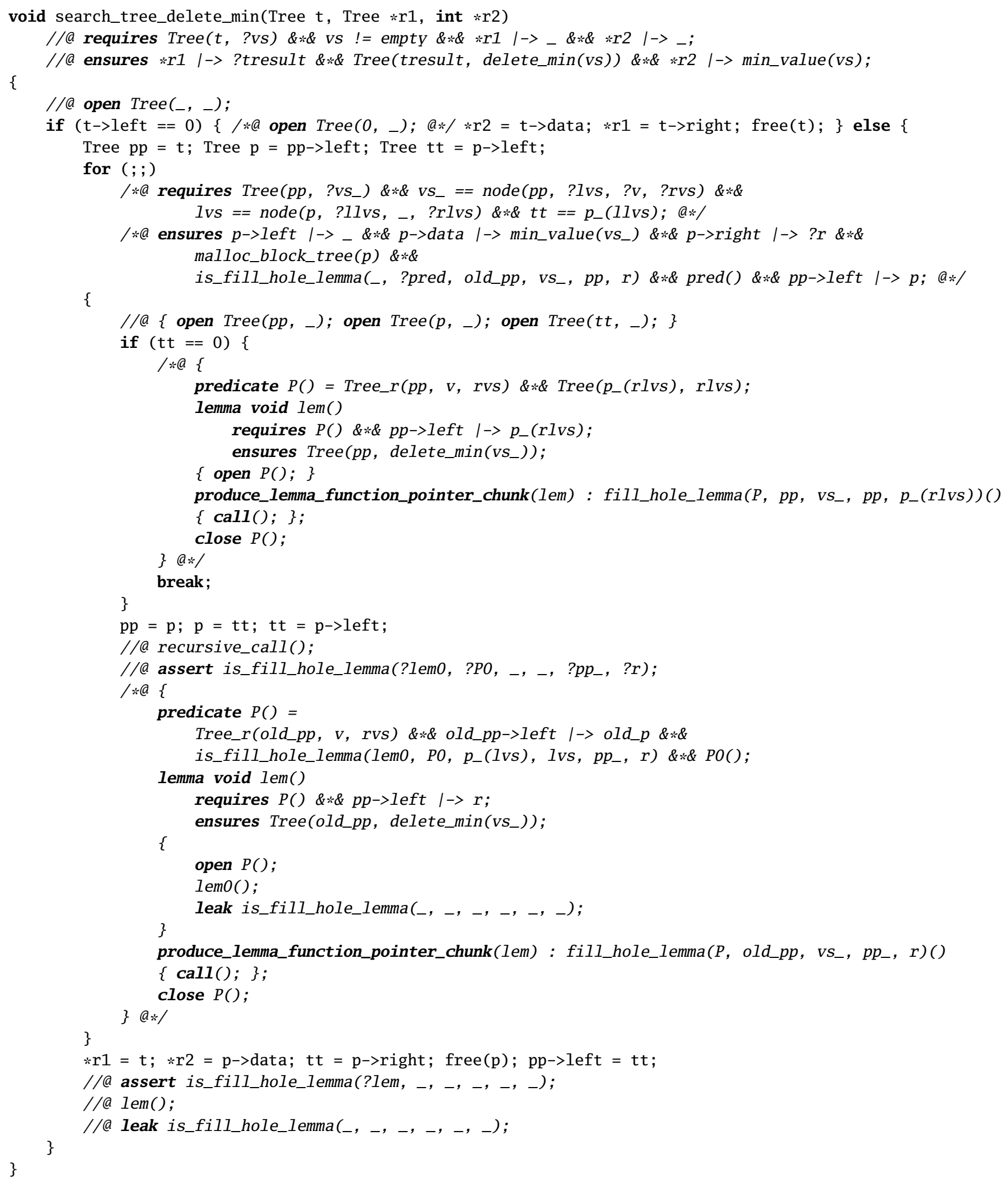

Fig. 8. Our competition solution for Challenge 3 (Part 2 of 2) 
command produces a lemma function pointer chunk of the form is_lft $(l f p, v 1, \ldots, v N)$ where $l f p$ is a pointer to the lemma function. (Of course, since lemma functions do not really exist in memory at run time, the pointer is not really a memory address but is just a value that identifies the lemma function.) Once we have a lemma function pointer chunk, we can perform a lemma function pointer call:

assert is_lft( $(? \mathrm{lfp}, \ldots, \ldots, \ldots)$;

$\operatorname{lfp}(\mathrm{w} 1, \ldots, \mathrm{wM})$;

We first bind the lemma function pointer to ghost variable $1 \mathrm{fp}$ and then perform a function call through that pointer, passing the arguments for the lemma function parameters. When encountering a lemma function pointer call, VeriFast checks that a lemma function pointer chunk exists in the symbolic heap. It then uses the values for the lemma function type parameters found in the chunk and the values for the lemma function parameters found in the call itself to instantiate the lemma function type precondition and postcondition, and finally uses the resulting contract to verify the call (by consuming the precondition and then producing the postcondition).

Our challenge solution defines the parameterized lemma function type fill_hole_lemma. Our loop returns a pointer to a lemma function that implements this type, instantiated appropriately.

The loop is again specified using a loop contract (see our discussion of our competition solution for Challenge 1 above). A particular "call" of the loop operates on the subtree pointed to by the current value of pp: given such a subtree, it returns the permissions for the fields of the node to be deleted (sufficient to deallocate the node) as well as the permission to update the left field that needs to be updated, and a magic wand (the combination of a lemma function pointer call permission is_fill_hole_lemma( ...) and a function-specific permission pred() holding the specific permissions required by the lemma to perform its duty) that can be used after the update to obtain the Tree permission describing the properly updated subtree at the old value of pp.

The code after the loop simply needs to call the returned lemma. To do so, it first uses an assert statement to bind the lemma function pointer (which is the first argument of the lemma function pointer call permission) to ghost variable lem. After the call, we use the leak command to acknowledge that we are consciously leaking the lemma function pointer call permission since we will not be using it further; this keeps VeriFast from reporting a leak error.

The body of the loop consists of two cases: either we have found the node to be deleted, or we need to continue our descent. In the former case, we define a lemma lem and an accompanying predicate $P$ that reassembles the subtree at pp given the right-hand section of pp (i.e. everything except the left field and the left subtree, represented by predicate Tree_r) and the right-hand subtree of p. We then use a produce_lemma_function_pointer_chunk command to generate a lemma function pointer call permission. This command generates a proof obligation that the lemma's contract implies the lemma function type's contract, appropriately instantiated. Any ghost commands required to discharge this proof can be written before or after the call () ; statement inside the ghost code block accompanying the command.

If we have not yet found the node to be deleted, we update the loop variables and perform the imaginary recursive call of the loop. (Note: in our competition solution for Challenge 1, the recursive call was implicitly inserted at the end of the loop body. Here we specify it explicitly so that we can insert ghost commands after the recursive call to derive the caller's postcondition from the callee's postcondition.) After the recursive call, we take the magic wand lemo returned by the recursive call (which operates on the subtree at $p$ ) and use it to build a new magic wand lem that operates on the subtree at pp.

\subsection{Post-competition Solution}

During the competition, we worked on an alternative approach for the third challenge. However, we did not manage to fully work out this solution in the allotted time, and we completed it only after the competition. It is shown in Figures 7 (shared with our competition solution), 9, and 10 .

The loop in the function search_tree_delete_min iterates over the left pointers until it finds the leftmost node in the tree. The key idea of this solution is that in each iteration the entire tree can be decomposed into a "tree with a hole" at pp (describing the part of the tree up to, but not including, the node at pp and its descendants) and another, complete tree starting at pp. Indeed, the loop invariant of Figure 10 states exactly this property.

To describe trees with a hole, we introduce the predicate Tree_with_hole (shown in Figure 9). The body of this predicate is similar to the body of Tree of Figure 7 except that the recursion stops when $t$ equals hole (instead of when $t$ is zero). The fixpoint merge fills the hole in a tree $\mathrm{t} 1$ by plugging in another tree $\mathrm{t} 2$.

A single iteration of the loop moves pp, $\mathrm{p}$ and tt down one node in the tree. The lemma move_hole states that doing so preserves the loop invariant. After the loop, the leftmost node is deleted from the tree. The lemma plug_hole is then used to recombine the tree with a hole and the tree at pp into a single tree, as required by the postcondition.

The predicate Tree_with_hole was inspired by the predicate lseg which is commonly used in separation logic proofs about linked lists. $\operatorname{lseg}(n 1, \mathrm{n} 2$, vs) holds if there exists a sequence of nodes from $n 1$ up to (but not including) n2 which contains the data values vs. In other 


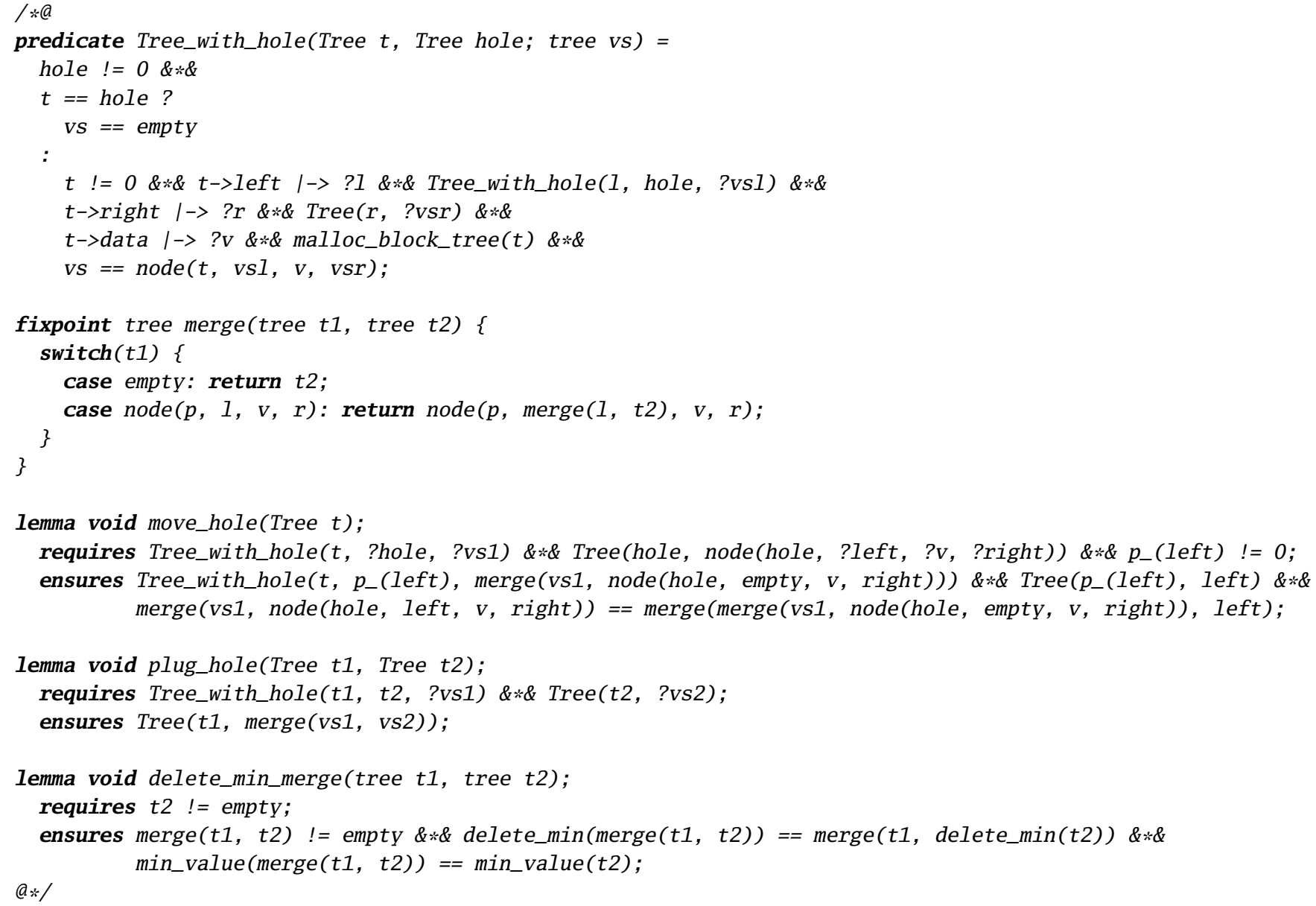

Fig. 9. Our post-competition solution for Challenge 3 (Part 1). The bodies of the lemmas have been omitted from this article.

words, lseg(n1, n2, vs) describes a linked list with a hole at n2.

The function contract of search_tree_delete_min shown in Figure 10 only states that the function deletes the leftmost node in the tree and that it returns the value stored in this node. In our actual implementation, we additionally prove that the resulting tree is a binary search tree, if the tree was a binary search tree on entry to the function.

\section{Conclusion}

For each of the three challenges issued during the competition, we were able to submit a solution. Moreover, the function contracts we came up with for each challenge are complete in the sense that they specify full functional correctness of the program at hand.

The annotation overhead and verification time required for each solution are shown in Table 1 . While the annotation overhead ranges from 3 up to 7 lines of annotations per line of code, each solution verifies in less than one second.
Table 1. Table showing the lines of code (LOC), lines of annotations (LOA), annotation overhead, and the verification time required for each solution. The experiments were executed on a MacBook Pro with a $2.53 \mathrm{GHz}$ Intel Core i5 processor and $4 \mathrm{~GB}$ of memory running Ubuntu Linux 12.04.1 LTS. The data in this table was gathered by running VeriFast from the command-line with the -stats option using Z3 [3] 2.3 to discharge proof obligations. Note that the lines of code and annotations only include the solution file itself; specification libraries that ship with VeriFast (such as list.gh) are not included in the measurements.

\begin{tabular}{lllll}
\hline solution & LOC & LOA & overhead & time \\
\hline LCP sol. 1 & 7 & 26 & $371 \%$ & $0.13 \mathrm{~s}$ \\
LCP sol. 2 & 5 & 16 & $320 \%$ & $0.03 \mathrm{~s}$ \\
PrefixSum & 29 & 194 & $669 \%$ & $0.28 \mathrm{~s}$ \\
TreeDel sol. 1 & 20 & 102 & $510 \%$ & $0.09 \mathrm{~s}$ \\
TreeDel sol. 2 & 18 & 114 & $633 \%$ & $0.09 \mathrm{~s}$ \\
\hline
\end{tabular}

In hindsight, we believe three key features of VeriFast enabled us to solve the challenges within the limited competition time frame: (1) the existing specification libraries, (2) fast verification and (3) separation logic. 


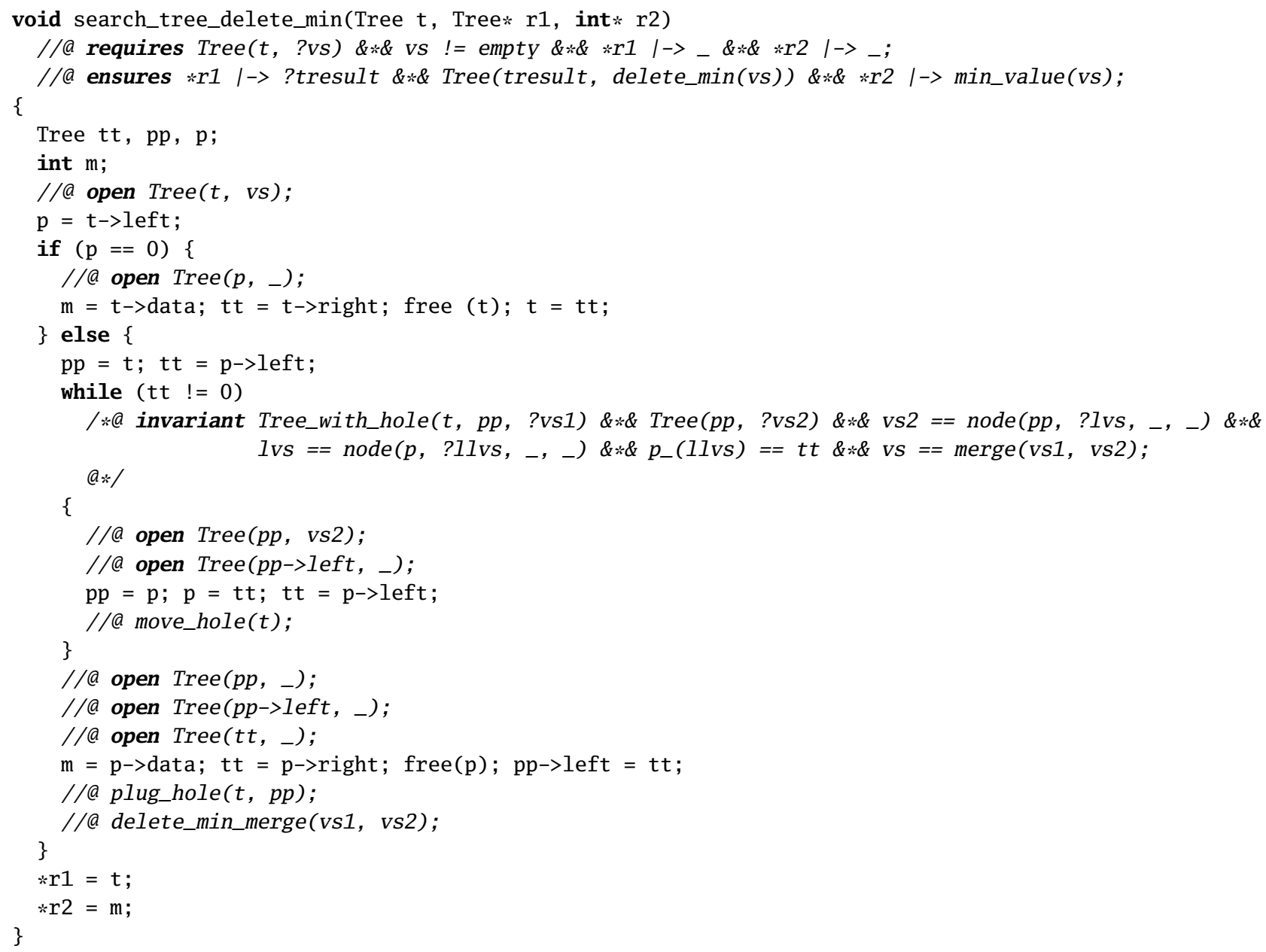

Fig. 10. Our post-competition solution for Challenge 3 (Part 2)

First of all, VeriFast ships with a number of specification libraries. In all our solutions, we reused definitions and lemmas provided by these libraries. For example, list.gh defines the function drop and offers a number of lemmas such as drop_n_plus_one and drop_length about this function that we reused in our first solution to Challenge 1. Secondly, VeriFast typically offers feedback on a verification attempt in under a second. This allowed us to stay focussed on the particular sub-problem at hand without losing time waiting for an answer from the verifier. Finally, the third challenge involves specifying a function operating on a tree. Thanks to separation logic, we were able to concisely specify the structure of the tree. Moreover, separation logic allowed us to reason locally: if a code fragment demands access only to a particular sub-tree, client code can safely assume that the fragment does not invalidate properties that hold for contexts containing that sub-tree (provided the context is disjoint from the sub-tree).

In the future, we plan to extend the automation in VeriFast such that additional ghost statements (such as lemma calls and open/close statements) can be inferred to reduce the annotation overhead. A key challenge in designing such an extension is keeping verification times low. Also, any inference algorithm will be incomplete, so we will need to provide ways for the user to supply hints.

We have participated in two other verification competitions in the past: the First [8] and Second [4] Verified Software Competitions. We did as follows: in the first, we were tied with all other participants; in the second, we were not among the medalists: we ended up in "Rank 8" with a score of $570 / 600$. In the end, a team's result in a verification competition of course depends not only on the "quality" of the tool and the approach, but also on the "shape" that the team members are in at the time of the competition, and to a large extent on random factors (i.e. "luck"). Still, during this latest competition we benefited from new features recently introduced into VeriFast, most prominently constructor patterns (e.g. xs $==\operatorname{cons}\left({ }_{-}, \ldots\right)$ ) and local predicates (e.g. predicates $\mathrm{P}$ in Figure 8), which, while not theoretically extending the power of VeriFast's logic, saved us some time, typing, and brain cycles. 
We enjoyed the competition very much and from the ensuing discussions we learned a great deal about the other verification approaches; we gratefully acknowledge the significant efforts by the organizers to bring about this successful outcome.

\section{Acknowledgments}

This research is partially funded by the Research Fund KU Leuven, and by the EU FP7 project NESSoS. With the financial support from the Prevention of and Fight against Crime Programme of the European Union (BCCENTRE). Jan Smans is a postdoctoral fellow of the Fund for Scientific Research Flanders (FWO). We acknowledge support from Microsoft Research Cambridge as part of the Verified Software Initiative.

\section{References}

1. Yves Bertot and Pierre Castéran. Interactive Theorem Proving and Program Development: Coq'Art: The Calculus of Inductive Constructions. Springer, 2004.

2. Richard Bornat, Cristiano Calcagno, Peter O'Hearn, and Matthew Parkinson. Permission accounting in separation logic. In POPL, 2005.

3. Leonardo de Moura and Nikolaj Bjørner. Z3: An efficient smt solver. In C. R. Ramakrishnan and Jakob Rehof, editors, TACAS, volume 4963 of Lecture Notes in Computer Science, pages 337-340. Springer, 2008.

4. Jean-Christophe Filliâtre, Andrei Paskevich, and Aaron Stump. The 2nd verified software competition: Experience report. In Vladimir Klebanov and Sarah Grebing, editors, COMPARE2012: 1st International Workshop on Comparative Empirical Evaluation of Reasoning Systems, Manchester, UK, June 2012. EasyChair.

5. C.A.R. Hoare. An axiomatic basis for computer programming. CACM, 12(10), 1969.

6. Marieke Huisman, Vladimir Klebanov, and Rosemary Monahan. The VerifyThis 2012 website. http://fm2012.verifythis.org/, 2012.

7. Bart Jacobs, Jan Smans, and Frank Piessens. The VeriFast website. http://distrinet.cs.kuleuven.be/software/VeriFast/, 2013.

8. Vladimir Klebanov, Peter Müller, Natarajan Shankar, Gary T. Leavens, Valentin Wüstholz, Eyad Alkassar, Rob Arthan, Derek Bronish, Rod Chapman, Ernie Cohen, Mark Hillebrand, Bart Jacobs, K. Rustan M. Leino, Rosemary Monahan, Frank Piessens, Nadia Polikarpova, Tom Ridge, Jan Smans, Stephan Tobies, Thomas Tuerk, Mattias Ulbrich, and Benjamin Weiß. The 1st Verified Software Competition: Experience report. In Michael Butler and Wolfram Schulte, editors, Proceedings, 17th International Symposium on Formal Methods (FM), volume 6664 of $L N C S$. Springer, 2011.

9. Tobias Nipkow. Interactive proof: Introduction to Isabelle/HOL. In O. Grumberg, T. Nipkow, and B. Hauptmann, editors, Software Safety and Security, pages 254 285. IOS Press, 2012.
10. Peter O'Hearn, John Reynolds, and Hongseok Yang. Local reasoning about programs that alter data structures. In $C S L, 2001$.

11. Matthew Parkinson and Gavin Bierman. Separation logic and abstraction. In POPL, 2005.

12. Pieter Philippaerts, Jan Tobias Mühlberg, Willem Penninckx, Jan Smans, Bart Jacobs, and Frank Piessens. Software verification with VeriFast: Industrial case studies. Science of Computer Programming, 2013.

13. John C. Reynolds. Separation logic: A logic for shared mutable data structures. In LICS, 2002.

14. Thomas Tuerk. Local reasoning about while-loops. In VSTTE Theory Workshop, 2010. 